\title{
Concomitant calciphylaxis and COVID-19 associated thrombotic retiform purpura
}

\author{
Jessica A. Rotman ${ }^{1,2} \cdot$ Kathryn E. Dean ${ }^{1,2} \cdot$ Cynthia Magro ${ }^{1,3} \cdot$ Gerard Nuovo $^{4} \cdot$ Roger J. Bartolotta $^{1,2}$
}

Received: 20 May 2020 / Revised: 1 August 2020 / Accepted: 9 August 2020 / Published online: 26 August 2020

(C) ISS 2020

\begin{abstract}
While initial reports regarding coronavirus disease 2019 (COVID-19) focused on its pulmonary manifestations, more recent literature describes multisystem abnormalities related to its associated microvascular angiopathy. Calciphylaxis is a rare systemic condition characterized by tissue necrosis in the setting of systemic microvascular calcifications. Both COVID-19 and calciphylaxis are procoagulant diagnoses associated with vascular-mediated cutaneous findings. To our knowledge, this is the first report to document the coexistence of COVID-19 associated retiform thrombotic purpura and calciphylaxis in a single patient, to link the pathologic etiologies of the two entities, and to describe the concomitant diagnoses' associated radiologic findings.
\end{abstract}

Keywords COVID-19 · Calciphylaxis $\cdot$ CT $\cdot$ Ultrasound $\cdot$ Panniculitis

\section{Introduction}

In addition to diffuse alveolar damage, which can be seen in the setting of viral pneumonia, complement-mediated microvascular injury and thrombosis play a critical role in the pathogenesis of acute respiratory distress syndrome associated with COVID19 [1]. Microangiopathy also plays a crucial part in the

Jessica A. Rotman

jar9120@med.cornell.edu

Kathryn E. Dean

ked9042@med.cornell.edu

Cynthia Magro

cym2003@med.cornell.edu

Gerard Nuovo

nuovo.1@osu.edu

Roger J. Bartolotta

rob9074@med.cornell.edu

1 Weill Cornell Medical College, 1300 York Avenue, New York, NY 10065, USA

2 Department of Radiology, Weill Cornell Medicine, 525 E. 68th Street, New York, NY 10065, USA

3 Department of Pathology and Laboratory Medicine, Weill Cornell Medicine, 1300 York Avenue, New York, NY 10065, USA

4 OSU Comprehensive Cancer Center, Columbus, Ohio 43210, USA development of extrapulmonary manifestations associated with COVID-19, including cutaneous retiform purpura, stroke, myocardial insufficiency, and acute renal failure [1].

Calciphylaxis (calcific uremic arteriolopathy) is a rare systemic condition that predominantly manifests as skin necrosis attributable to subcutaneous thrombotic calcific arteriopathy and microangiopathy. In the pathogenesis of calciphylaxis, there is a critical role for a bone-forming phenotype within the vasculature as well as a procoagulant state for triggering arterial intimal and microvascular adluminal calcification [1-4].

We report a case of an ischemic dermopathy syndrome developing in the setting of COVID-19 exhibiting hybrid overlapping features of retiform thrombotic purpura and calciphylaxis. The pathophysiology that underlies this distinctive presentation along with the associated radiologic findings is presented. By recognizing the ultrasound and CT appearance of the vasculopathy-mediated cutaneous manifestations of these systemic disorders, radiologists can play an essential role in their diagnosis prior to biopsy and expedite treatment to prevent clinical deterioration.

\section{Case report}

A 62-year-old female with end-stage renal disease secondary to focal segmental glomerulosclerosis (recently initiated on 
peritoneal dialysis), rheumatoid arthritis (on Humira and prednisone), hypertension, type II diabetes, and well-controlled hypothyroidism (on Synthroid) presented to the emergency department with 3 weeks of cough and atraumatic bilateral leg pain, which was associated with tense edema and red discoloration.

In the emergency department, she was afebrile and tachycardic with normal oxygen saturation on room air. Her initial labs were remarkable only for hyponatremia secondary to a missed prior dialysis session.

Her physical exam was notable for firm edema and erythema about both knees, greatest near the popliteal fossae, with masslike areas of indurated dusky plaques. Hyperpigmentation and xerosis were also noted in the non-edematous portions of the more distal lower extremities.

Lower extremity venous duplex ultrasound was negative for deep venous thrombosis. Additional targeted sonographic evaluation of the bilateral popliteal soft tissues demonstrated thin linear echogenic foci with associated shadowing, suspicious for microcalcifications (Fig. 1a). In addition, there were heterogeneous nodular hyperechoic areas within the edematous subcutaneous fat with associated mild mass effect on the underlying fascia, consistent with panniculitis (Fig. 1b). There was no evidence of hyperemia to suggest cellulitis (Fig. 1c).

Upon admission, the patient was found to be COVID-19 positive via SARS-CoV-2 RT-PCR. CT angiography was also obtained given concern for an ischemic etiology of the patient's pain and cutaneous abnormalities. CT demonstrated heterogeneous foci of nodular and reticular edema scattered throughout the subcutaneous fat of the medial distal thighs (Fig. 2), compatible with panniculitis. Additionally, scattered netlike fine branching calcifications were noted in the subcutaneous fat. The angiogram component demonstrated scattered dense atherosclerotic calcifications but no evidence of high-grade stenosis.
The patient's lower extremity edema and hyponatremia improved after hemodialysis and fluid restriction. However, the thigh pain progressed and the rashes became more violaceous and nodular, which prompted inpatient dermatology consultation. The retiform purpura plaques of the bilateral lower extremities (left greater than right) were clinically concerning for calciphylaxis given the patient's chronic prednisone use, end-stage renal disease, and secondary hyperparathyroidism. A punch biopsy of the left medial thigh plaque was obtained.

The skin biopsy demonstrated striking vascular alterations throughout the dermis and subcutaneous fat. Within the skin overlying the fat, there was focal full-thickness epidermal necrosis as well as marked necrosis of the adnexal structures, including hair follicles, eccrine ducts, and glands of the eccrine coil. There were occlusive luminal thrombi and focal mural fibrin deposition. In association with a thrombotic microangiopathy, these findings indicated that the epithelial necrosis was of ischemic etiology. Within the subcutaneous fat, there was also a thrombotic diathesis with a dominant pattern of localization to capillaries and venules (Fig. 3a). There was concomitant ischemic-driven lipomembranous fat necrosis.

A calcific microangiopathy was also noted, with granular basophilic deposits of calcium seen within the capillaries. The calcium was visualized with a von Kassa stain (Fig. 3b). There were striking deposits of $\mathrm{C} 3 \mathrm{~d}, \mathrm{C} 4 \mathrm{~d}, \mathrm{C} 5 \mathrm{~b}-9$, and MASP2 within the microvasculature of the dermis (Fig. 3c) and subcutaneous fat, indicative of complement activation.

Immunohistochemical assessment for the SARS-CoV-2 envelope protein (Fig. 3d) and ACE2 receptor (Fig. 3e) revealed focal weak endothelial cell staining amidst superficial dermal vessels and very strong endothelial cell staining in the deep dermis and subcutaneous fat microvasculature. Neither viral protein nor ACE2 receptor expression was identified in the endothelia of the thrombosed vessels. The constellation of clinical, imaging, and histopathologic findings indicated a
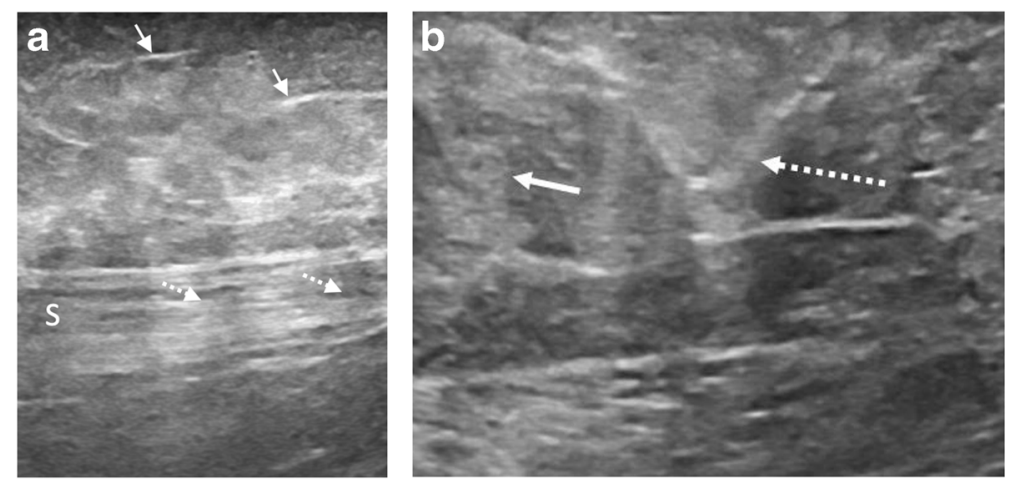

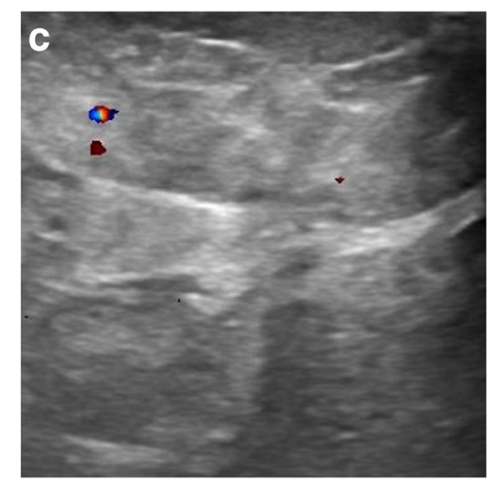

echogenic foci secondary to nodular edema within the subcutaneous fat (arrow) with mild mass effect on the interlobular septa (dashed arrow). Transverse color Doppler image (c) from the same region lacks hyperemia to suggest cellulitis 
Fig. 2 Bilateral lower extremity CT angiography. Axial (a, b) and coronal (c) post-contrast CT images demonstrate netlike branching superficial microvascular calcifications (arrows), multifocal reticulonodular edema (dashed arrows) within the subcutaneous fat of the medial distal thighs, and scattered triangular areas of cutaneous edema (arrowheads) along the margins of the panniculitis
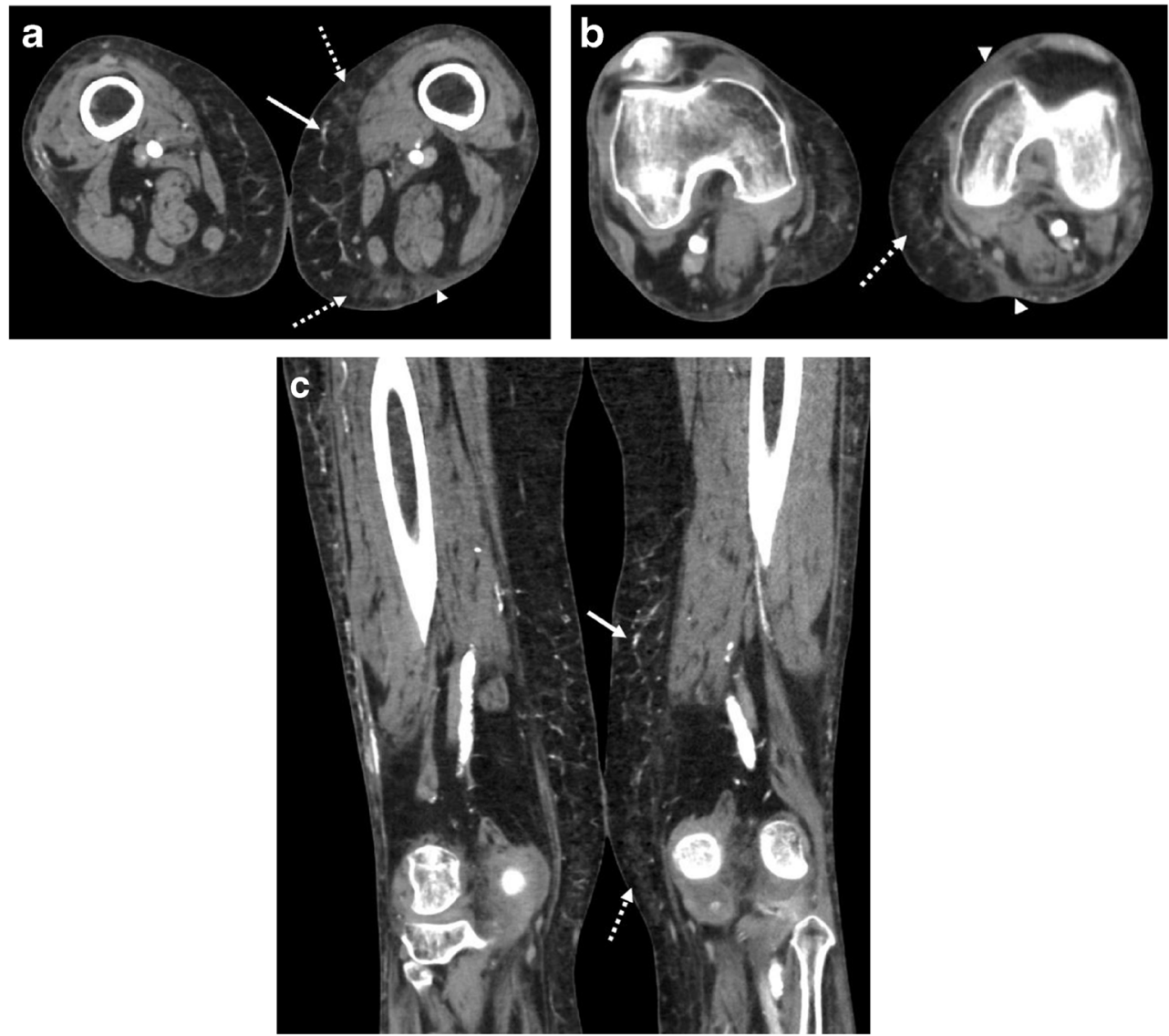

hybrid of overlapping COVID-19 associated retiform thrombotic purpura and calciphylaxis.

The patient's calciphylaxis improved upon dialysis optimization and the administration of Sensipar and sodium thiosulfate. However, her leg pain continued and she was given lidocaine patches and placed on a tramadol PCA pump. Initially requiring $2 \mathrm{~L}$ oxygen by nasal cannula after admission, she was weaned to room air and completed a course of hydroxychloroquine for COVID-19. After clinical stabilization 15 days into admission, the patient was discharged to a nursing home with an oral dilaudid prescription for her leg pain and plans for outpatient follow-up with dermatology. Four weeks after discharge, she was readmitted with diarrhea and fluctuating mental status. During the course of the admission, the patient developed worsening anemia and new thrombocytopenia felt to represent gastrointestinal blood loss and potentially heparin-induced thrombocytopenia. With a DNR/ DNI in place, the patient passed away 5 days after admission.

\section{Discussion}

While the most common symptoms of COVID-19 include fever, dry cough, dyspnea, malaise, and new loss of taste or smell, clinical presentations vary widely, ranging from asymptomatic silent infection to multi-organ failure [5-7]. Nearly $20 \%$ of patients infected with COVID-19 present with severe disease characterized by microangiopathic ARDS as well as extrapulmonary thrombotic complications associated with markedly elevated D-dimers, indicative of excessive activation of the coagulation pathway $[1,8]$.

The vast majority of COVID-19 radiology literature to date has focused on the pulmonary manifestations of the disease with findings on chest $\mathrm{CT}$ including multifocal peripheral ground glass opacities, consolidations, and septal thickening [9-11]. The first report of cutaneous manifestations of COVID-19 [12] described erythematous rashes, widespread urticaria, and chickenpox-like vesicles predominantly involving the trunk. A few cases of transient livedo reticularis (a netlike pattern of reddish to blue skin discoloration related to vasospasm throughout the cutaneous microvasculature) have also been reported in association with COVID-19 [13].

In our case of COVID-19 associated thrombotic retiform purpura, there was a pauci-inflammatory thrombogenic vasculopathy associated with extensive deposits of $\mathrm{C} 3 \mathrm{~d}, \mathrm{C} 4 \mathrm{~d}$, C5b-9, and MASP2 within the microvasculature, which is diagnostic of activation of the mannan lectin and alternative pathways. The presence of extensive SARS-CoV-2 protein within the endothelium of the pulmonary and cutaneous vasculature was previously established as the potential basis of 

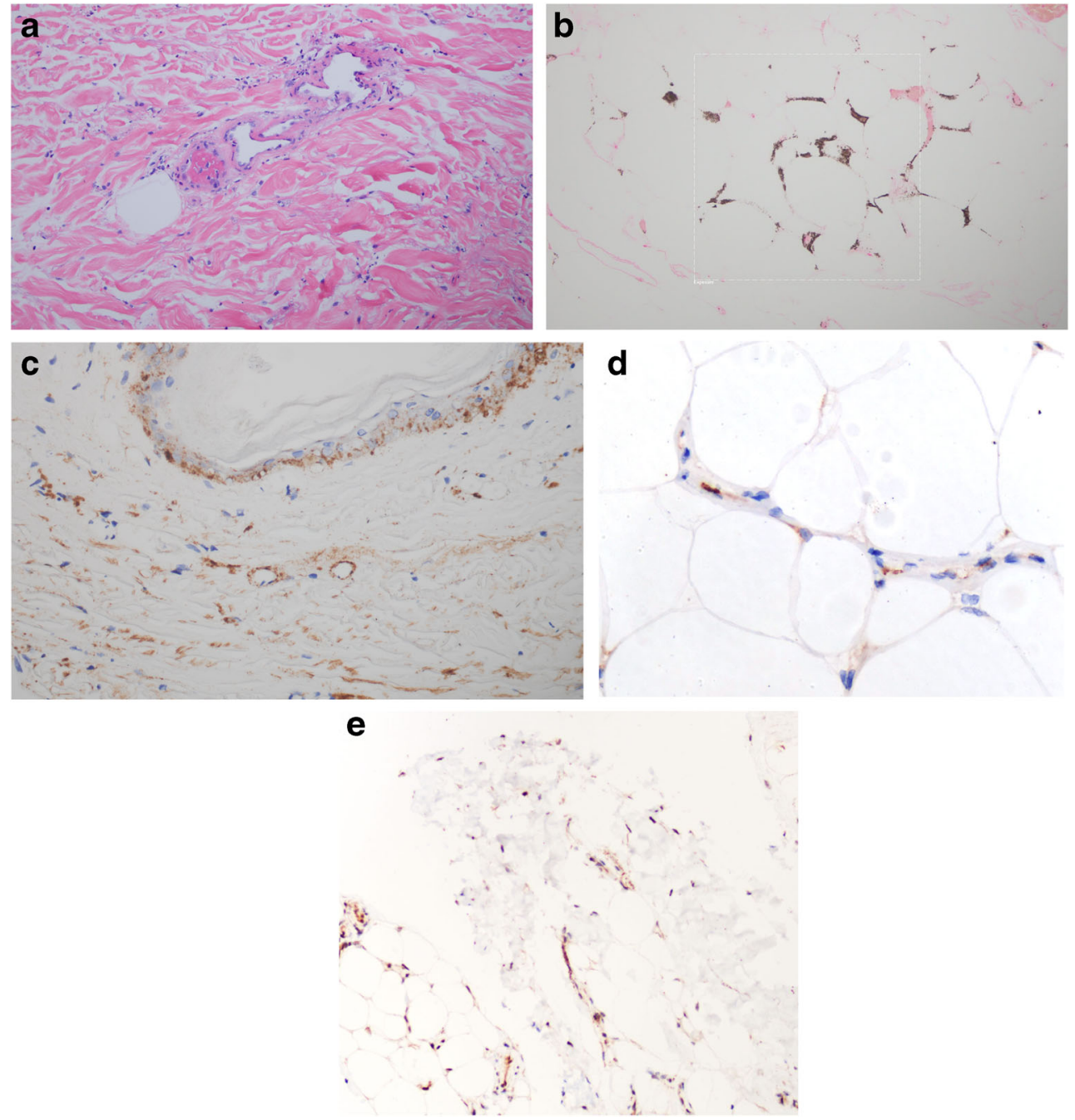

Fig. 3 In situ deposition of components of complement activation (C3d, C4d, C5b-9, and MASP2). Assays were conducted on $4 \mu \mathrm{m}$ sections procured from formalin-fixed paraffin-embedded tissue. Immunohistochemical assessment of SARS-CoV-2 associated envelope protein and ACE2 receptor expression was also conducted. A pauciinflammatory thrombogenic vasculopathy was present throughout the dermis (a) and subcutaneous fat (hematoxylin and eosin, $\times 400$ ). A Von Kassa preparation confirmed the presence of calcium within the subcutaneous microvasculature (b) (Von Kassa, $\times 400)$. A critical role for complement activation in the pathogenesis of the thrombotic alterations was revealed by the extent of $\mathrm{C} 4 \mathrm{~d}$ and $\mathrm{C} 5 \mathrm{~b}-9$ deposition. Illustrated are granular deposits of C5-9 within the microvasculature of the dermis (c) (diaminobenzidene, $\times 400$ ). Endothelial viral microparticles were confirmed via immunohistochemical assessment for the presence of SARS$\mathrm{CoV}-2$ envelope protein (primary antibodies from ProSci Poway, CA at 1:500 dilution, $\times 200)(\mathbf{d})$ as well as ACE2 receptor (primary antibodies from Proteintech at a 1:13,000 dilution, $\times 1000)(\mathbf{e})$ within the deep dermis and subcutaneous fat the mannan lectin pathway activation, with the spike glycoprotein likely the critical impetus to complement activation in severe COVID-19 [1]. In our case, biopsy specimens were extensively positive for SARS-CoV-2 envelope/spike glycoprotein and ACE2 receptors within the endothelia of the microvasculature in the deep dermis and subcutaneous fat.

Calciphylaxis (or calcific uremic arteriolopathy) is a rare, highly morbid systemic condition characterized by microvascular calcifications leading to tissue ischemia and necrosis. Patients with calciphylaxis typically present with violaceous skin discolorations, intense pain, and ultimately cutaneous ulceration. Our patient had multiple risk factors for the development of calciphylaxis, including end-stage renal disease with secondary hyperparathyroidism, peritoneal dialysis, female gender, autoimmune disease, chronic immunosuppressant medications, and diabetes $[2-4,14-18]$.

The current gold standard for establishing the diagnosis of calciphylaxis is skin biopsy [19-21]. The biopsy findings in calciphylaxis are highly reproducible. This distinct ischemic dermopathy syndrome shows striking microvascular and arteriopathic alterations that are confined to the subcutaneous fat without similar changes in the overlying dermis. The subcuticular alterations include a distinctive form of obliterative calcific intimal expansion by calcium and collagen leading to marked vascular attenuation [2-4]. The caliber of 
affected vessels is in the 220 to 500 micron size range. In addition, the capillaries of the fat show a pauci-inflammatory thrombogenic vasculopathy with adluminal and endothelial cell calcification [2-4]. In this particular case, the subcutaneous changes were typical for calciphylaxis with the dominant pathology being a subcuticular pauci-inflammatory thrombotic diathesis affecting capillaries and venules with supervening microvascular calcification, including calcific endothelial cell mummification. At variance with typical findings, however, were the overlying dermal changes, which are not seen in calciphylaxis. These dermal changes were typical for COVID-19 associated retiform purpura, as revealed by the presence of bland luminal thrombi within the microvasculature and striking associated complement localization [1, 21].

Due to its rarity and nonspecific presentation, the diagnosis of calciphylaxis is often delayed. In cases of pending or unavailable biopsy specimens, radiologic imaging can aid in rapid diagnosis of calciphylaxis [20]. Calciphylaxis can manifest on Tc-99m MDP bone scintigraphy as asymmetric radiotracer uptake in the subcutaneous tissues, most intensely in areas corresponding to skin plaques and nodules [22-28]. Radiographically, mildly increased soft tissue density with a netlike pattern of calcification has been demonstrated in patients with calciphylaxis $[19,29,30]$. Unlike calcific arteriosclerosis, this classic netlike pattern involves small- and medium-sized vessels and is due to the perpendicular configuration of dermal and subcutaneous central arterioles with blood vessels in the fascia [30]. The sonographic appearance of calciphylaxis has been described as numerous linear branching echogenic foci with posterior acoustic shadowing scattered throughout the superficial soft tissues $[18,28]$, typically deposited in adipose rich sites of the lower extremities (less commonly involving the trunk, and rarely the face or upper extremities) [30].

Since calciphylaxis can present with initial symptoms similar to those of lower extremity deep venous thrombosis, Doppler ultrasound may be the first examination to demonstrate the soft tissue microvascular calcifications of calciphylaxis [28]. In addition to these microvascular calcifications, the ultrasound in this case also revealed heterogeneous nodular foci of increased echogenicity throughout the subcutaneous fat with mild mass effect on the underlying fasciae, suspicious for panniculitis, which is another common imaging feature of calciphylaxis [21]. The CT angiogram corroborated this diagnosis, demonstrating reticulonodular edema in the involved subcutaneous fat with triangular areas of cutaneous edema along the margins of the panniculitis. The small branching vessel calcifications and densely calcified yet widely patent main lower extremity arteries further mirrored the histologic findings.

The extensive intravascular fibrin deposition, which is mediated by complement activation in severely ill COVID-19 patients, could induce a bone-forming phenotype through activation of osteopontin within the subcuticular vessels. Hence, the perfect microenvironmental milieu for calciphylaxis is present in patients suffering from severe COVID-19 [2-4]. Given the procoagulant state and various overlapping risk factors of the two entities, in addition to the postulated relationship between elevated fibrin and osteopontin activation, we suspect that more cases demonstrating concurrent COVID-19 associated thrombotic retiform purpura and calciphylaxis will be reported in the future.

\section{Conclusion}

This case is unique due to the hybrid overlapping features of calciphylaxis and COVID-19 associated thrombotic retiform purpura, both captured in a single biopsy specimen and demonstrated on ultrasound and CT. This case highlights the potential for the development of calciphylaxis as an additional manifestation of severe COVID-19 illness and highlights the importance of recognizing its characteristic imaging, as radiologists may be the first to suggest the diagnosis, even before ulceration is apparent on clinical examination. Given this newfound association and the poor prognosis and high mortality rates associated with calciphylaxis and advanced cases of COVID-19, radiologic imaging can be a crucial aid in diagnosis and necessary timely clinical management.

\section{References}

1. Magro C, Mulvey JJ, Berlin D, Nuovo G, Salvatore S, Harp J, et al. Complement associated microvascular injury and thrombosis in the pathogenesis of severe COVID-19 infection: a report of five cases. Transl Res. 2020;15.

2. Magro CM, Momtahen S, Hagen JW. Osteopontin expression in biopsies of calciphylaxis. Eur J Dermatol. 2015;25(1):20-5.

3. Magro CM, Simman R, Jackson S. Calciphylaxis: a review. J Am Col Certif Wound Spec. 2010;2(4):66-72.

4. Wilmer WA, Magro CM. Calciphylaxis: emerging concepts in prevention, diagnosis, and treatment. Semin Dial. 2002;15(3):172-86.

5. Guan WJ, Ni ZY, Hu Y, Liang WH, Ou CQ, He JX, et al. Clinical characteristics of coronavirus disease 2019 in China. N Engl J Med. 2020;382(18):1708-20.

6. Zhou F, Yu T, Du R, Fan G, Liu Y, Liu Z, et al. Clinical course and risk factors for mortality of adult inpatients with COVID-19 in Wuhan, China: a retrospective cohort study. Lancet. 2020;395(10229):1054-62.

7. Wang $\mathrm{D}, \mathrm{Hu} \mathrm{B}, \mathrm{Hu} \mathrm{C}$, Zhu F, Liu X, Zhang J, et al. Clinical characteristics of 138 hospitalized patients with 2019 novel coronavirus-infected pneumonia in Wuhan. China JAMA. 2020.

8. Zhai Z, Li C, Chen Y, Gerotziafas G, Zhang Z, Wan J, et al. Prevention and treatment of venous thromboembolism associated with coronavirus disease 2019 infection: a consensus statement before guidelines. Thromb Haemost. 2020.

9. Ye Z, Zhang Y, Wang Y, Huang Z, Song B. Chest CT manifestations of new coronavirus disease 2019 (COVID-19): a pictorial review. Eur Radiol. 2020. 
10. Chung M, Bernheim A, Mei X, Zhang N, Huang M, Zeng X, et al. CT imaging features of 2019 novel coronavirus (2019-nCoV). Radiology. 2020;295(1):202-7.

11. Kanne JP, Chest CT. Findings in 2019 novel coronavirus (2019$\mathrm{nCoV}$ ) infections from Wuhan, China: key points for the radiologist. Radiology. 2020;295(1):16-7.

12. Recalcati S. Cutaneous manifestations in COVID-19: a first perspective. J Eur Acad Dermatol Venereol. 2020.

13. Manalo IF, Smith MK, Cheeley J, Jacobs R. A dermatologic manifestation of COVID-19: transient livedo reticularis. J Am Acad Dermatol. 2020.

14. Zhou Q, Neubauer J, Kern JS, Grotz W, Walz G, Huber TB. Calciphylaxis. Lancet. 2014;383(9922):1067.

15. Smith S, Inaba A, Murphy J, Campbell G, Toms AP. A case report: radiological findings in an unusual case of calciphylaxis 16 years after renal transplantation. Skelet Radiol. 2013;42(11):1623-6.

16. Weenig RH, Sewell LD, Davis MD, McCarthy JT, Pittelkow MR. Calciphylaxis: natural history, risk factor analysis, and outcome. J Am Acad Dermatol. 2007;56(4):569-79.

17. Fine A, Zacharias J. Calciphylaxis is usually non-ulcerating: risk factors, outcome and therapy. Kidney Int. 2002;61(6):2210-7.

18. Bukhman R, Scheri RP, Selim MA, Baker JA. Sonography in the identification of calciphylaxis of the breast. J Ultrasound Med. 2010;29(1):129-33.

19. Halasz CL, Munger DP, Frimmer H, Dicorato M, Wainwright S, Calciphylaxis: comparison of radiologic imaging and histopathology. J Am Acad Dermatol. 2017;77(2):241-6.e3.

20. Bleibel W, Hazar B, Herman R. A case report comparing various radiological tests in the diagnosis of calcific uremic arteriolopathy. Am J Kidney Dis. 2006;48(4):659-61.

21. Nigwekar SU, Thadhani R, Brandenburg VM. Calciphylaxis. N Engl J Med. 2018;378(18):1704-14.
22. Raduka J, Aggarwal A, Johnson K, Byun K, Trace AP. Bone scintigraphy findings in calciphylaxis. Radiol Case Rep. 2018;13(2): 315-9.

23. Itani M, Matesan M, Behnia S, Elojeimy S. Calciphylaxis on bone scan: correlation between molecular and cross-sectional findings. Radiol Case Rep. 2017;12(1):175-8.

24. Gripp MJ, Gagliardi JA. Calciphylaxis on technetium bone scan: two case reports. Radiol Case Rep. 2007;2(2):30-2.

25. Han MM, Pang J, Shinkai K, Franc B, Hawkins R, Aparici CM. Calciphylaxis and bone scintigraphy: case report with histological confirmation and review of the literature. Ann Nucl Med. 2007;21(4):235-8.

26. Paul S, Rabito CA, Vedak P, Nigwekar SU, Kroshinsky D. The role of bone scintigraphy in the diagnosis of calciphylaxis. JAMA Dermatol. 2017;153(1):101-3.

27. Norris B, Vaysman V, Line BR. Bone scintigraphy of calciphylaxis: a syndrome of vascular calcification and skin necrosis. Clin Nucl Med. 2005;30(11):725-7.

28. Bonchak JG, Park KK, Vethanayagamony T, Sheikh MM, Winterfield LS. Calciphylaxis: a case series and the role of radiology in diagnosis. Int J Dermatol. 2016;55(5):e275-9.

29. Shmidt E, Murthy NS, Knudsen JM, Weenig RH, Jacobs MA, Starnes AM, et al. Net-like pattern of calcification on plain softtissue radiographs in patients with calciphylaxis. J Am Acad Dermatol. 2012;67(6):1296-301.

30. Baby D, Upadhyay M, Joseph MD, Asopa SJ, Choudhury BK, Rajguru JP, et al. Calciphylaxis and its diagnosis: a review. J Family Med Prim Care. 2019;8(9):2763-7.

Publisher's note Springer Nature remains neutral with regard to jurisdictional claims in published maps and institutional affiliations. 